\title{
APORTACIÓN AL ESTUDIO DEL MOLINO HIDRÁULICO EN LA CASTILLA MEDIEVAL: LOS MOLINOS DEL MONASTERIO DE SANTA CLARA DE ALCOCER*
}

\author{
Pablo Martín Prieto \\ Universidad de Alcalá de Henares
}

RESUMEN: El presente artículo aborda la descripción de un caso particular de cierto interés en la evolución de los equipamientos molineros en la Castilla medieval, como es el de los molinos que en la Edad Media formaron parte del patrimonio del monasterio de clarisas de la población alcarreña de Alcocer. De la descripción de las condiciones técnicas y socioeconómicas que rodean la realidad de estos molinos de las clarisas de Alcocer se siguen interesantes conclusiones sobre la implantación social del molino bidráulico en la región en la Edad Media, al tiempo que se logran algunas precisiones útiles para la caracterización de la economía agraria de la zona.

Palabras Clave: Molino hidráulico. Economía agraria. Alcocer. Técnica. Clarisas.

ABSTRACT: This paper focuses on a particular example in the medieval evolution of the watermill in Castile, that of the medieval watermills belonging to the patrimony of a Poor Clares monastery in the village of Alcocer, in the Castillan region of the Alcarria. From the description both of the technical and socio-economic circumstances of the Poor Clares watermills, we can draw interesting conclusions about the social implantation of medieval watermills in the same area, as well as some useful details about the development of the region's agrarian economy.

KeY WORDS: Watermill. Agrarian economy. Alcocer. Technique. Poor Clares.

* Abreviaturas: AHN: Archivo Histórico Nacional, Madrid. AMA: Archivo Municipal de Alcocer, Alcocer (Guadalajara). 
En el presente trabajo pretendemos realizar una contribución a la comprensión de la importancia socioeconómica del molino hidráulico en el desarrollo de la economía agraria castellana medieval, centrándonos en un caso concreto de cierto interés, el que atañe a los molinos que el monasterio de Santa Clara de Alcocer integró en su patrimonio durante la Edad Media.

En otro lugar ${ }^{1}$ tenemos realizado un estudio completo de la realidad medieval del monasterio clariso de Alcocer, cuya fundación fue emprendida, a mediados del siglo XIII, como resultado de una iniciativa concertada entre la corona castellana y la familia nobiliaria de los Guzmán. La creación en 1255 del señorío de Alcocer, Cifuentes, Viana y Palazuelos para la que fuera amante de Alfonso X, doña Mayor Guillén de Guzmán, señorío vinculado expresamente por el rey Sabio a la continuidad dinástica en la línea de su descendencia bastarda que comienza en Beatriz, hija de ambos amantes y luego reina de Portugal, se halla en el origen del impulso regio y nobiliario a la fundación clarisa de Alcocer, las primeras seis décadas de cuya trayectoria estarán especialmente ligadas a esa rama alfonsina de los Guzmán².

La imposición de un modelo organizativo basado en la experiencia de los monasterios benedictinos aseguró la integración de un patrimonio monástico amplio, en el que las rentas de molinos desempeñaron desde el mismo comienzo un papel central. En la segunda década del siglo XIV, la familia de Guzmán renunció al señorío de Alcocer. A partir de ese momento, la comunidad clarisa disfrutó, por la fuerza de los hechos, de una casi irrestricta independencia respecto de los nuevos señores de la villa que se fueron sucediendo a lo largo de los siglos XIV y XV. En uso de esta notable autonomía de gestión de sus destinos y de su propia política patrimonial, las clarisas de Alcocer intensificaron, de manera consciente y programada, la dedicación del monasterio a las rentas y aprovechamientos molineros, capítulo del patrimonio monástico que en todo momento parece haber sido objeto de una atención preferente.

Abordamos en las siguientes páginas, en primer lugar, una descripción pormenorizada de todos los molinos que durante la Edad Media, en un momento o en otro, formaron parte del patrimonio monástico de las clarisas de Alcocer, prestando especial atención al aspecto físico y técnico de los mismos. Pasamos a continuación revista a las coordenadas principales del marco jurídico al que en la Edad Media hubo de ceñirse la edificación y explotación de estos ingenios. El análisis de la realidad de los mismos no quedaría completo sin referirse a las implicaciones de orden socioeconómico, referidas principalmente a las modalidades de propiedad y explotación, así como a precisiones sobre la rentabilidad de los molinos, con las que se cierra nuestro estudio.

\footnotetext{
1 Martín PRieto, Pablo: El monasterio de Santa Clara de Alcocer en la Edad Media, Guadalajara, 2005.

2 Sobre la formación y trayectoria de este señorío en manos de la rama alfonsina de los Guzmán: MARTín PRIETO, Pablo: «Origen, evolución y destino del señorío creado para la descendencia de Alfonso X de Castilla y Mayor Guillén de Guzmán (1255-1312)», Temas Medievales (Buenos Aires) 11 (2002-2003) 219-240.
} 


\section{DESCRIPCIÓN DE LOS MOLINOS DEL MONASTERIO CLARISO DE ALCOCER.}

Entrando a describir pormenorizadamente los molinos cuyas rentas formaron parte del patrimonio monástico de las clarisas de Alcocer durante la Edad Media, lo primero que cabe observar es que la documentación no permite establecer con seguridad, para cada uno de ellos, si se trataba de ingenios de tipo horizontal o vertical, aunque parece adecuado suponer que en la mayoría o en la práctica totalidad de los casos se trató de molinos de tipo vertical: en primer lugar, por las características de los cursos de agua sobre los que aparecen instalados; en segundo lugar, por la dilatada tradición de infraestructuras y aprovechamientos hidráulicos que parece posible seguir en la zona, quizás aun desde antes de la conquista cristiana del territorio; en tercer lugar, por la amplia difusión que este tipo de molino había alcanzado, con carácter general, aun desde antes del comienzo del periodo de referencia, a mediados del siglo XIII.

Abordamos en primer lugar los complejos de molinos radicados en el término de Alcocer y sus inmediaciones. El actual término de Alcocer ocupa 6.207 hectáreas entre los $40^{\circ} 30^{\prime}$ y los $40^{\circ} 26^{\prime}$ de latitud y entre el $1^{\circ} 02^{\prime}$ y el $1^{\circ} 10^{\prime}$ de longitud (meridiano de Greenwich). No existe constancia de que se haya producido variación histórica significativa alguna en los límites de dicho término, razón que nos impulsa a aceptar en lo fundamental, para el propósito de nuestra investigación, la precedente localización geográfica. El término de Alcocer se encuentra comprendido en la comarca de la Alcarria Baja, en el centro de la depresión hoy conocida como Hoya del Infantado, formada a su paso por la región por el curso del río Guadiela, uno de los afluentes del Tajo. El término limita al norte con los términos de Casasana y Millana; al sur, con el curso del río Guadiela, al otro lado del cual están los términos de Cañaveruelas, Alcohujate y Castejón; al este, con los términos de Salmeroncillos y Villar del Infantado (antes llamado Villar de Ladrón); al oeste, con el término de Córcoles. El paisaje presenta una característica combinación de fértiles valles de origen fluvial con moderadas irregularidades de terreno y algunos arroyos naturales (no todos llevan agua todo el año ${ }^{3}$ ) que van a verter, ya en el curso del Guadiela, ya en el del arroyo que pasa por Salmerón y cruza el término de Alcocer, de norte a sur, hasta aportar en el Guadiela: arroyo que en las fuentes manejadas recibe el nombre de Riato o Regato de San Miguel (pues atravesaba el antiguo término de la aldea despoblada de San Miguel del Monte), y que hoy se llama Garigay.

La mayor concentración de molinos hidráulicos en los que las clarisas de Alcocer tienen intereses se localiza precisamente en el referido Riato del término despoblado de San Miguel, que desde el primer momento formó parte del patrimonio del monasterio. En la dotación fundacional de Mayor Guillén de

3 Como sugiere la aparición del topónimo menor "Arroyo Seco» en el ítem n ${ }^{\circ} 65$ de un apeamiento de 1519: AHN, Clero, libro 4140, ff. 122r-132v. 
septiembre de 1260 , los molinos representan una parte muy significada del conjunto de bienes y rentas que la comunidad recibió como base para la constitución de su patrimonio monástico. El montante global de la renta anual derivada de los derechos de explotación de molinos que el convento recibió en esta ocasión asciende a 100 cahíces de cereal. De esta suma, una parte importante se corresponde con las rentas generadas por cuatro complejos de molinos situados en el cauce del Riato ${ }^{4}$ :

Los molinos de Millán «el Gordo», y los de Domingo Martín, sendos complejos adquiridos en fecha imprecisa por Mayor Guillén y cedidos en $1260 \mathrm{al}$ monasterio clariso. El nombre con que se conocen remite a la iniciativa privada de unos propietarios particulares en el origen de estos complejos hidráulicos, si bien no cabe establecer mayores precisiones sobre sus características materiales, ni sobre la trayectoria seguida antes de su integración en el patrimonio del monasterio clariso.

Otras dos paradas de molinos en el Riato, situadas la una aguas arriba y la otra aguas abajo del monasterio clariso, mandadas ambas edificar por Mayor Guillén, con vistas a su integración en el patrimonio del monasterio.

Algunas décadas más tarde, a fin de completar el conjunto de dotaciones de infraestructuras hidráulicas situadas en la inmediación del mismo complejo monástico, la comunidad, de propia iniciativa, mandará edificar, a distintos particulares, algunas obras adicionales que en adelante quedarán incorporadas al patrimonio del monasterio.

En primer lugar, en 1323 el convento concluye con Juan Martín, un operario cualificado, pues consta su condición de molinero de la vecina localidad de Valdeolivas, un contrato para la edificación de una presa y un caz en el Riato, junto al edificio monástico, para las necesidades de agua de la comunidad'. Estas necesidades pueden relacionarse con labores de regadío de las huertas que las monjas mantenían en la inmediación de la casa monástica para abastecimiento alimentario propio. La presa fue reparada por el mismo Juan Martín en 1331, al término de un periodo de ocho años en que, siguiendo las previsiones contractuales, había disfrutado del usufructo de dicha infraestructura ${ }^{6}$.

En segundo lugar, en 1327 la comunidad encargó a su paniaguado Domingo Máñez de Beteta la construcción de otras dos casas de molinos nutridas por las aguas del Riato, en la inmediación del complejo monástico, concluida una antes de 1327 y la otra poco después de esa fecha ${ }^{7}$; casi con toda seguridad, estas dos casas de molinos se edificaron sobre el caz acondicionado en 1323 por el molinero Juan Martín, pues una de las condiciones especificadas en el contrato suscrito con el paniaguado Domingo Máñez es la de construir una

\footnotetext{
4 AHN, Clero, carpeta 566, doc. no 4 (en adelante, 566/4).

AHN, Clero, 567/7.

6 AHN, Clero, 567/14.

AHN, Clero, 567/10.
} 
presa aguas arriba del caz, para servicio de estas dos nuevas casas de molinos. Para conseguir la regularidad de abastecimiento del caz, se acomete asimismo la construcción de una buharda o vano de piedra, en arco, en el arranque del caz, y de una tapia de argamasa encima del mismo vano, para impedir que en caso de crecida entrara en el caz más de un volumen regulado (por el arco) de agua del Riato. Otra particularidad de interés concierne a la inclusión, en cada una de estas dos casas de molinos, de sendos establos capaces de albergar cada uno hasta tres bestias de carga, destinadas muy probablemente a ayudar en las tareas asociadas a la explotación y mantenimiento de los molinos, tales como labores de transporte de los granos molidos, o de la leña que se precisara. Por añadidura, se menciona expresamente que, a estas dos casas de molinos con establos incorporados, se vincula una huerta asociada, de una aranzada de superficie, que el mismo Domingo Máñez debería plantar de árboles frutales. Es un ejemplo particularmente interesante de las sinergias que se establecen entre las labores de molturación de grano y las de regadío, aprovechando una misma dotación de infraestructura hidráulica: el agua del caz movía los molinos y proporcionaba regadío para la huerta de frutales, en una asociación muy habitual, que veremos repetirse en otros casos, en el patrimonio de las clarisas de Alcocer, y que, adicionalmente, parece apoyar la caracterización de este complejo como molinos de tipo vertical, que permiten un servicio simultáneo de regadío.

Aguas abajo, cerca ya del punto donde el Riato confluye en el río Guadiela, se situaban otros dos importantes complejos de molinos sobre los que las clarisas de Alcocer obtuvieron derechos. Los intereses del convento fueron adquiridos a partir de una operación de permuta de ciertos bienes llevada a cabo en 1299 por la entonces abadesa del monasterio, Mencía Pérez Carrillo, con Pedro Vicente, clérigo y hombre de confianza de la infanta Blanca de Portugal, a la sazón señora de Alcocer ${ }^{8}$. El primero de los dos complejos de molinos que eran propiedad de dicho clérigo y que a partir de 1299 se integran en el patrimonio monástico de las clarisas son los molinos llamados de Aparicio Martínez, por el que fuera su anterior propietario, tal vez su constructor. Se encontraban situados en la horcajadura entre el Guadiela y el Riato de San Miguel, y tenían una haza o huerta asociada.

El segundo es el importante complejo de molinos que fue y se llamó de Donato, en la misma confluencia del Guadiela y del Riato. Se trataba de una importante infraestructura de molinos harineros, que asimismo incorporaba un batán, y una haza o huerta de regadío servida por un mismo caz que tomaba agua del Guadiela. Consta que, al menos en 1301, las monjas ordenaron a los arrendatarios del molino ejecutar algunas mejoras y reparaciones, consistentes en «afondar el caz» y erigir una pared de argamasa de 20 brazadas de largo, 3 de alto y una de ancho, destinada probablemente a asegurar la regularidad del abastecimiento del caz, separando su trazado del curso del río. Sobre la complejidad

8 AHN, Sellos, 55/14. 
técnica de este conjunto de molinos y batán, es testimonio la utilización en las fuentes de la época de términos como «fierros», «rodeznos» y «marranos», que documentan la utilización de mecanismos de hierro9. De la importancia de este complejo hidráulico para la economía local y monástica da idea el hecho de que se haya procedido a incesantes reparaciones y ampliaciones técnicas, llegando a incrementar regularmente el número de ruedas harineras. Así, por un inventario de bienes del monasterio clariso, sabemos que en 1337 el complejo de Donato constaba ya de cuatro ruedas harineras y un batán ${ }^{10}$. Dos años más tarde, en 1339, pasaba a constar de cinco ruedas harineras y el batán. Este último documento proporciona algunos interesantes detalles adicionales de orden material: las paredes de la casa de molinos eran de argamasa; la presa que regulaba la entrada de agua del caz estaba edificada en piedra; y las muelas, soleras y correderas, tenían unas medidas de un palmo y una mano de alto, y seis palmos de diámetro ${ }^{11}$.

Le toca ahora el turno a los complejos hidráulicos dependientes, en sentido estricto, del Guadiela, río caudaloso y de indudable importancia económica para toda la comarca ${ }^{12}$. Se tiene noticia, en primer lugar, de unos molinos comprados por Mayor Guillén y cedidos al monasterio clariso en 1260, sin localización precisa ${ }^{13}$. Pero la más relevante infraestructura hidráulica del curso del Guadiela eran tal vez los molinos situados junto al puente, un hito capital en la organización de los tráficos de la actividad humana y económica en la zona, con el que se conectaban importantes caminos de la época, y sobre el que discurría el tráfico de los ganados trashumantes de la cañada real de Aragón. La construcción de estos molinos, sobre la que nada puede precisarse, se debía muy probablemente a la iniciativa privada. En 1402 eran propiedad de la familia Martínez: de tres ruedas que constaban, las rentas de dos de ellas nutrían una capellanía fundada por Catalina Martínez; la restante la tenía su hermano Juan Martínez, quien en la fecha referida cedió sus derechos sobre los molinos del puente a la por entonces señora de Alcocer, Constanza de Villena, en una operación de permuta ${ }^{14}$. Con posterioridad, la misma Constanza de Villena donó, por legado testamentario, estos derechos al monasterio clariso, que se hizo con la plena propiedad en 1428. Incidentalmente, por la toma de posesión del molino que en esta última fecha hicieron los representantes legales del monasterio clariso, sabemos de la persona del molinero titular, Alfonso Garrido, y del detalle de que las puertas del complejo podían cerrarse con cerrojo $^{15}$. Se trataba de un

9 AHN, Clero, 567/2.

10 AHN, Clero, 567/17 y 567/18.

11 AHN, Clero, 567/19.

12 Por el ancho curso de este cauce sabemos que se transportaban troncos de árbol seccionados con destino a la construcción, entre otros testimonios, por un inventario de los bienes del mayorazgo del Infantado de 1570: AHN, Osuna, legajo 3410, f. 260v.

13 AHN, Clero, 566/4.

14 AHN, Clero, 568/9.

15 AHN, Clero, 569/2. 
complejo de molinos harineros instalado junto al puente, dotado de una presa que sabemos hubo necesidad de reparar en más de una ocasión, por causa de los desperfectos acarreados sobre la infraestructura por las crecidas del río ${ }^{16}$.

Siguiendo el curso del Guadiela, y ya en el término de Alcohujate, el monasterio clariso tenía derechos sobre los molinos llamados de Mari Cosida. Según el inventario de las propiedades del monasterio de 1337 , los primeros intereses de la comunidad sobre este complejo de molinos, concretamente sobre dos ruedas harineras, fueron incluidos en la primera donación de la fundadora Mayor Guillén, de $1260^{17}$. Con el tiempo, las clarisas adquirieron más derechos sobre el mismo complejo, ya fuera por compra o donación, ya encargando sucesivas obras y mejoras. Así, en 1328, nos encontramos con que el monasterio tiene ya derechos sobre tres ruedas harineras y un batán, que cede mediante un arrendamiento por tres años al ya conocido Juan Martín, molinero de Valdeolivas $^{18}$. Años más tarde, en 1337, los mismos molinos y batán se hallarán arrendados por cinco años, y rentan al monasterio doce cahíces y medio de trigo al año ${ }^{19}$. A cuenta de estos molinos, la documentación permite conocer algunas especificaciones técnicas, como las medidas de las muelas (nuevamente establecidas en un palmo y una mano de altura, y seis palmos de diámetro), o referentes a algunas herramientas vinculadas a su manejo, como una azuela, un «escepro» y dos picos. El «escepro» o «esceptro» era un cetro, esto es, una de las varas o perchas de la alcándara, tendido donde se colgaba ropa, más concretamente, el sayal que según las fuentes pisaba este batán ${ }^{20}$.

En el núcleo urbano de Alcocer, las monjas tuvieron la propiedad de un molino aceitero. Ya en el inventario de bienes del monasterio clariso de 1337 se mencionaba de pasada un molino de aceite, aunque sin precisar su localización ${ }^{21}$. En 1496 las clarisas establecerán un censo enfitéutico sobre su molino de aceite (no consta que sea el mismo que en 1337 tenían arrendado), del que se especifica su localización urbana y el aprovechamiento de agua que el ingenio precisaba, si bien, a falta de ulteriores informaciones, no podemos inferir por qué medio obtenía esas «servidunbres de agua» que atestigua la documentación ${ }^{22}$.

16 En varias respuestas proporcionados por los testigos en el curso de un interrogatorio judicial celebrado en 1572 con motivo de un pleito por la propiedad de estos molinos a que nos referimos, se deja constancia del hecho de que una presa aneja al puente, que daba servidumbre de agua al caz que nutría los referidos molinos, servía asimismo de protección para el puente, en caso de crecida o avenida, "por que la dicha presa es estribo della [de la puente] e reçibe el golpe e fuerça del agua quando biene de abenida» (AMA, libro A4, f. 63r), de manera que alguna vez «se la llevo el rio de una abenida o creçida» (AMA, libro A4, f. 65r).

17 AHN, Clero, 567/17 y 567/18.

18 AHN, Clero, 567/11.

19 AHN, Clero, 567/17 y 567/18.

20 AHN, Clero, 567/11.

21 AHN, Clero, 567/17 y 567/18.

22 La situación precisa es en el barrio o cuadrilla de Pareja, en el límite del casco de la población, lindando con una tierra de cultivo, con unas casas, con una calle pública y con unos 
Fuera de Alcocer y sus aledaños, el monasterio disfrutó también de algunos relevantes intereses sobre molinos. Una mención temprana pero sin continuidad en la documentación presenta una renta de 50 maravedíes anuales donada a la comunidad en 1260 por el hermano de la fundadora, situada en unos molinos de su propiedad, en Murcia ${ }^{23}$. Del destino de esta renta en moneda nada más se sabe, y no consta que el monasterio la cobrara, por ejemplo, en el inventario de 1337: más bien parece que la lejanía de la renta dificultó su percepción o fue causa de que la comunidad la perdiera, con compensación o sin ella.

Más cerca de Alcocer, el monasterio retuvo derechos sobre algunos molinos situados en Cifuentes y su entorno. El origen de los intereses del monasterio clariso de Alcocer en molinos de Cifuentes y su tierra debe entenderse vinculado con el hecho de que la fundadora del convento, Mayor Guillén de Guzmán, fuera simultáneamente titular del señorío tanto de Alcocer como de Cifuentes. En su dotación fundacional de 1260, Mayor Guillén cedió a las clarisas de Alcocer la participación que como titular del señorío de Cifuentes ella poseía en los molinos concejiles llamados de la Balsa, que constituyen sin duda el más importante complejo molinero de Cifuentes ${ }^{24}$. La parte de estos molinos cuyos derechos fueron percibidos por el monasterio de Alcocer ascendía en 1337 a ocho ruedas harineras, y la rentabilidad se fija entonces en 36 cahíces de trigo anuales ${ }^{25}$. Mayor Guillén estableció los derechos de molienda en el cinco por ciento del grano molido $^{26}$, provisión que fue confirmada por sucesivos señores de Cifuentes ${ }^{27}$.

Por ser propios del señorío, la explotación de estos molinos de la Balsa de Cifuentes comportaba algunos elementos propios de un régimen de monopolio. Según parece, la primera señora de Alcocer y de Cifuentes, Mayor Guillén, había acordado con el concejo de Cifuentes rebajar las tasas de pesos y fanegas que como señora debía percibir en esta villa, a cambio de la sujeción de los vecinos de Cifuentes a prácticas de monopolio relativas a los molinos señoriales: así, se vedaba a los vecinos de Cifuentes la posesión de molinos, e incluso el llevar su grano a moler a otros molinos distintos de los señoriales de la villa; se concedía derechos ilimitados de aprovechamiento de leña en todo el monte de Cifuentes para servicio de estos molinos; los vecinos asumían la obligación de mantener limpio el río de donde los molinos obtenían el agua; y se comprometían a acarrear a costa del concejo las muelas que los molinos precisaran substituir ${ }^{28}$.

pajares. Lo que se cede en censo es la «casa de molinos de aceyte, con las servidunbres de agua, e viga, e ruedas, e calderas, e casares». AHN, Clero, legajo 1967.

${ }^{23}$ AHN, Sellos, 55/4.

24 AHN, Clero, 566/4.

25 AHN, Clero, 567/17 y 567/18.

26 La maquila a veinte supone la entrega por el servicio de una parte de cada veinte del grano molido. Sobre esta maquila fija a veinte en Cifuentes, cfr. LAYNA SERRANO, Francisco: Historia de Cifuentes, Guadalajara, 1979, $2^{\mathrm{a}}$ ed., apéndice III, p. 268.

27 Como su hija la reina Beatriz de Portugal, en 1272: AHN, Clero, 566/12 y 566/13.

28 Todas estas condiciones son pormenorizadas en AHN, Clero, 566/12 y 566/13. 
El mismo régimen de corte monopolístico se aplica expresamente, al menos desde 1272, a la parte señorial de los molinos llamados de Ordoño que poseyó el monasterio clariso de Alcocer. El complejo molinero de Ordoño se localiza en el término de Gárgoles de Arriba, aldea de Cifuentes; parece haber tenido cierta relevancia en la economía de la zona, contando con un número de particioneros entre los que se repartía la propiedad. Mayor Guillén adquirió, probablemente como aneja al señorío de Cifuentes, una parte en las rentas de estos molinos, que en 1260 donó al monasterio clariso de Alcocer: sobre esta participación señorial se estableció el mismo tipo de restricciones monopolísticas que estaban vigentes sobre los de la Balsa. En 1272, la reina Beatriz de Portugal, señora de Alcocer y de Cifuentes como heredera de su madre Mayor Guillén, dispuso que las mismas condiciones señoriales se aplicasen a las partes de que el monasterio clariso de Alcocer pudiera apropiarse en lo sucesivo, en estos mismos molinos de Ordoño ${ }^{29}$. De esta suerte, se abrían las puertas para la incorporación por parte del monasterio, en el mismo régimen de virtual monopolio, de mayores parcelas de la propiedad del complejo. Dado que los vecinos de Cifuentes y su aldea el Val de San García quedaban obligados a llevar a moler su grano a estos molinos señoriales propiedad de las monjas de Alcocer, tanto a los de la Balsa en la misma villa como a los de Ordoño en la cuesta de Gárgoles de Arriba, parece razonable suponer que la rentabilidad de otros ingenios molineros que en la zona subsistieran en manos de otros propietarios acabaría restringida drásticamente a los términos más modestos de la economía familiar. En efecto, los propietarios independientes de molinos en la zona, así como los particioneros del molino de Ordoño una vez que fue afectado por los usos señoriales, debieron de sucumbir por la falta de clientes y acabarían vendiendo sus derechos molineros. Un ejemplo bien documentado de este proceso lo encontramos en 1285, cuando Domingo Lázaro de la Fuente vende a la abadesa del monasterio clariso de Alcocer la novena parte de la propiedad del molino de Ordoño, «con todo el derecho», por 50 maravedíes ${ }^{30}$. No queda constancia de otras transacciones similares, pero es de suponer que el monasterio clariso de Alcocer, como depositario de los derechos señoriales sobre estos molinos, acabara completando la plena propiedad sobre los mismos.

En las inmediaciones de este complejo de Ordoño, entre Gárgoles y Cifuentes, consta que las clarisas de Alcocer dispusieron de intereses sobre cuando menos otros dos complejos molineros. En 1335, el monasterio de Alcocer recibió la donación de una casa de molinos con dos ruedas harineras y un batán, situada «en la parada de molinos que dizen de la Cuesta, que son cerca de Çifuentes, en termino de Atiença», esto es, poco más o menos en el mismo paraje

29 «Et si por aventura mas molinos pudier aver este monesterio en aquel termino do es el de Ordonno sobre dicho, muelan [los vecinos de Cifuentes y Val de San García] a ellos»: AHN, Clero, 566/13.

30 AHN, Clero, 566/17. 
del molino de Ordoño ${ }^{31}$. La mención al término de Atienza no permite descartar en todo caso que este complejo se hallara, como el de Ordoño, en término de Gárgoles de Arriba, que pertenecía a la tierra de Atienza. En el texto de la donación, tanto la mención al batán como a los «fierros» que intervienen en su construcción prueban que se trata, también en este caso, de ingenios de tipo vertical. Sobre el mismo río que lleva de Gárgoles a Cifuentes, pero ya en el término de esta última localidad, en el paraje llamado de la Fuente Rodera, sabemos que las clarisas de Alcocer tuvieron una rueda de molino harinero desde fecha indeterminada, pero en todo caso anterior a la de 1438, en que la ceden en censo al concejo de Cifuentes ${ }^{32}$. En consecuencia, en torno al área de Cifuentes deben incluirse todos estos intereses molineros en el complejo de Ordoño, en otros molinos de la Cuesta de Gárgoles de Arriba, y en el mismo término de Cifuentes.

\section{El marco legal.}

Dejando por el momento de lado esa temprana mención a unas rentas de molinos situadas en Murcia, sobre la que carecemos de toda otra noticia posterior a su donación ${ }^{33}$, a los propósitos de nuestra investigación tan sólo le cumple alcanzar por el momento alguna precisión sobre los molinos localizados en los respectivos ámbitos de influencia de Cifuentes y de Alcocer, que representan la inmensa mayoría de los intereses en molinos del monasterio clariso de Alcocer.

Sabemos que Cifuentes, luego de su segregación del alfoz de Atienza para integrarse en el señorío creado por Alfonso X para su antigua amante Mayor Guillén en $1255^{34}$, recibió de esta su primera señora el privilegio de retener el fuero de Atienza ${ }^{35}$. Desgraciadamente, sobre el fuero de Atienza no cabe alcanzar gran precisión, ya que su texto no ha llegado hasta nosotros. En lo relativo al capítulo de molinos, dada su temprana redacción, no cabe esperar que haya sido especialmente prolijo en reglamentaciones, de manera que podemos aceptar sin otra alteración, en lo que concierne a los molinos de Cifuentes y de Gárgoles de Arriba, el marco general de la legislación castellana relativa a molinos.

La política de Mayor Guillén siguió el mismo camino en el caso de Alcocer, población a la que, tras su segregación de la tierra de Huete para formar parte del nuevo señorío, concedió conservar el fuero de su antigua cabeza de alfoz ${ }^{36}$. El fuero de Huete es una adaptación del fuero de Cuenca, a propósito del cual

31 AHN, Clero, 567/16.

32 AHN, Clero, legajos 1965 y 1967.

33 AHN, Sellos, 55/4.

34 MARTÍN PRIETO: «Origen, evolución y destino del señorío», p. 221.

35 Como consta por la confirmación de dicho fuero otorgada por su sucesora Beatriz en 1281: Archivo Municipal de Cifuentes, caja 71, doc. $\mathrm{n}^{\circ} 4$.

36 Martín Prieto: «Origen, evolución y destino del señorío», p. 224, nota 12. 
se ha afirmado, acaso forzando un punto de exageración al respecto, que en su texto, de todos los aspectos de la producción agraria, sólo las técnicas hidráulicas no quedan por entero en la obscuridad ${ }^{37}$. A continuación pasaremos breve revista a los aspectos sobre molinos legislados en el fuero de Huete.

En primer lugar es necesario referirse a las disposiciones del fuero tendentes a asegurar los dos grandes principios conectados que regulan la realidad del molino hidráulico: la libertad en el realengo y la prevalencia del derecho de antigüedad. En el fuero se concede expresa libertad para poner molinos hidráulicos en funcionamiento en los cauces fluviales ${ }^{38}$, incluso si se trata de instalarlos en un caz abierto por un tercero ${ }^{39}$, con la sola restricción del derecho de antigüedad, que impide perjudicar a un propietario de molinos o presas ya establecido con una infraestructura nueva ${ }^{40}$, y que permite al constructor de un caz escoger en el mismo el mejor lugar para instalar sus propios molinos ${ }^{41}$. Como resultado de la afirmación de este principio-guía de antigüedad, se estipula que cualquier molino, presa o caz que se instalara en perjuicio de otros más antiguos debería ser destruido en el plazo de tres días tras el juicio; con todo, en este punto, las dos versiones del fuero presentan una diferencia reveladora, relativa a la multa que el infractor debería satisfacer por cada día de incumplimiento de esta disposición: 10 maravedíes al día según el texto del fuero de Cuenca, tan sólo 5 maravedíes en el fuero de Huete: esta diferencia puede ser tenida por significativo indicio de la importancia económica relativa de los molinos e infraestructuras hidráulicos en los respectivos ámbitos de aplicación de los dos fueros, esto es, en las áreas económicas de Cuenca y Huete respectivamente ${ }^{42}$.

Otras disposiciones forales se destinan a garantizar la paz del molino, castigando con fuertes penas los atentados contra la integridad de los molinos, bienes sujetos a una especial protección por parte del derecho público. El allanamiento de molino se equipara en todo al allanamiento de morada ${ }^{43}$, se prevén severas penas contra los incendiarios de molinos $^{44}$, y se fijan indemnizaciones para compensar los daños ocasionados en los equipamientos hidráulicos: 10 maravedíes en el fuero de Cuenca ( 5 en el fuero de Huete), por romper una muela, una rueda o un canal, y 10 maravedíes más el doble del daño causado

37 GAUTIER-DALCHÉ: «Formes et organisation de la vie rurale dans le Fuero de Cuenca», en Cuenca y su territorio en la Edad Media (Actas del I Simposio Internacional de Historia de Cuenca), MadridBarcelona, 1982, p. 164.

38 Fuero de Huete, en Martín Palma, María Teresa: Los fueros de Villaescusa de Haro y Huete, Málaga, Universidad, 1984 (en adelante, F. Huete), rúbrica 121; y Fuero de Cuenca, en UREÑA Y SMenJAud, Rafael (ed.): Fuero de Cuenca, Madrid, 1935 (en adelante, F. Cuenca), cap. VIII, rúbrica 2.

39 F. Huete, 121; F. Cuenca, VIII, 5.

40 F. Huete, 121; F. Cuenca, VIII, 3-6.

41 F. Huete, 121; F. Cuenca, VIII, 6.

42 F. Huete, 130; F. Cuenca, VIII, 18.

43 F. Huete, 127, 133; F. Cuenca, VIII, 14, 22.

44 F. Huete, 125; F. Cuenca, VIII, 13. 
en el fuero de Cuenca ( 5 y el daño doblado en el fuero de Huete) por inutilizar ruedas de aceña, baño, huerto o pozo ${ }^{45}$.

Algunas reglamentaciones conciernen aspectos técnicos sobre la erección y mantenimiento de los equipamientos hidráulicos. Así, en el fuero de Cuenca se estipula con claridad que cada casa de molinos debería respetar un espacio en derredor de nueve pasos y contar con un camino de acceso de un mínimo de tres pasos de anchura; en el fuero de Huete, por el contrario, se omiten estas especificaciones, declarándose tan sólo que los nuevos molinos «ayan tales entradas e tales salidas quantas fasta aqui ouieron»: de esta forma, el texto del fuero de Huete respeta las condiciones físicas de los molinos existentes, sin imponer condiciones tan precisas como el fuero de Cuenca. Este detalle, unido a los ejemplos ya antes mencionados de menor valoración económica de los equipamientos hidráulicos, da nuevamente idea de la menor importancia de éstos en la tierra de Huete, respecto de los de la tierra de Cuenca ${ }^{46}$. Otros aspectos técnicos contemplan la obligación del constructor de un caz de levantar puentes para salvarlo, allí donde el concejo competente dispusiera ${ }^{47}$; y la obligación de los beneficiarios de regadíos, que sin duda se extendía a los propietarios de caces, de hacerse cargo de la limpieza de las lindes de las acequias. La multa por no hacerlo es de dos maravedíes por semana en el fuero de Cuenca, y de tan sólo uno en el de Huete ${ }^{48}$.

Un detalle significativo permite cobrar mejor conciencia de la importancia de la protección que el derecho público dispensaba a los molinos como infraestructura de carácter estratégico, cuando se regula el caso de los molinos falsos o simulados, levantados por sus propietarios defraudadores para beneficiarse del especial tratamiento que el poder concedía a los verdaderos y productivos ${ }^{49}$.

Otro capítulo se refiere a conflictos por el uso de las aguas, en tres casos: entre molinos; entre un molino y un regadío; entre un molino y tierras de labor. En el primer caso se contempla la posibilidad de que un molino situado aguas abajo de un cauce pudiera perjudicar a otro situado aguas arriba del mismo, por la mala regulación del nivel de agua que depende de la presa del primero de estos ingenios: la multa que el propietario del molino responsable de esa mala regulación del nivel debería satisfacer al propietario del molino perjudicado nuevamente dobla, en el caso del fuero de Cuenca, la cantidad estipulada en el fuero de Huete ${ }^{50}$. En el segundo caso, se dispone que cuando los cultivos de regadío necesitaran del agua de un curso, río o caz, empleado en dar servicio a molinos, la pudieran tomar, los martes y viernes de cada semana,

\footnotetext{
45 F. Huete, 128, 129; F. Cuenca, VIII, 15, 16.

46 F. Huete, 121; F. Cuenca, VIII, 1.

47 F. Huete, 121; F. Cuenca, VIII, 7.

48 F. Huete, 126; F. Cuenca, VIII, 12.

49 F. Huete, 122; F. Cuenca, VIII, 9.

50 F. Huete, 121; F. Cuenca, VIII, 8.
} 
en los puntos previstos por las autoridades locales ${ }^{51}$. En el tercer caso, se trata de los eventuales perjuicios ocasionados en tierras de labor por inundación o filtración de las aguas que dan servicio a un molino, una presa o un $\mathrm{caz}^{52}$.

Otras disposiciones regulan el régimen de copropiedad sobre molinos, estipulando que si uno de los particioneros se opusiera a trabajar en el molino contra el acuerdo de los restantes copropietarios, se vería obligado a pagarles una compensación por la parte proporcional de los gastos de explotación (en mano de obra y en reparaciones), bien en un pago monetario, bien a través de la requisa de la parte que le correspondiera de los beneficios del molino ${ }^{53}$.

Por último, se hace referencia a los derechos de explotación o régimen de maquilas, que se establecen, de ordinario, en un $5 \%$ del grano molturado (maquila a 20), y en el periodo estival, desde el día de San Juan hasta el de San Miguel, en un 6,7 \% (maquila a 15). De todos estos derechos de molienda, al molinero le pertenecía percibir una cuarta parte $^{54}$.

\section{EL RÉGIMEN DE PROPIEDAD Y EXPLOTACIÓN.}

Estos dos últimos aspectos regulados en el fuero nos conducen a la consideración del régimen de propiedad y explotación de los molinos de la zona. Santiago Aguadé ha establecido un esquema de validez general para el área de Cuenca y Huete, de acuerdo con el cual, sobre la base de la existencia de infraestructuras hidráulicas en la época de dominación musulmana, en los primeros tiempos de la repoblación cristiana la iniciativa de pequeños y medianos propietarios se revela crucial para la continuidad y reactivación de estos equipamientos y de su importancia económica en la región. A partir del siglo XIII, observa Aguadé un proceso paulatino de concentración de la propiedad de los molinos y otros equipamientos hidráulicos, en las manos de grandes propietarios, muchos de ellos eclesiásticos ${ }^{55}$. Con todo, Sánchez Benito ha advertido del peligro de exagerar, como si se tratara de una verdadera «señorialización» a escala sistemática, este proceso de concentración en manos de grandes propietarios de la propiedad de los molinos, un proceso que sin duda tuvo lugar, pero cuyo alcance conocido se debe en parte a un sesgo introducido por la índole de las fuentes disponibles, y que en todo caso no impidió el mantenimiento de la que él llama "propiedad popular» de los molinos durante todo el siglo XIV ${ }^{56}$.

51 F. Huete, 131; F. Cuenca, VIII, 19.

52 F. Huete, 123; F. Cuenca, VIII, 10.

53 F. Huete, 124; F. Cuenca, VIII, 11.

54 F. Huete, 132; F. Cuenca, VIII, 20, 21.

55 AguAdÉ NieTo, Santiago: «Notas para el estudio del molino hidráulico en Cuenca (11771300)», Almud (1982) 47-80 [70-72].

56 SÁNCHeZ BENITO, José María: Las tierras de Cuenca y Huete en el siglo XIV. Historia económica, Cuenca, 1994, pp. 108-109. 
Este último autor hace referencia, como hecho excepcional, al caso de un carpintero que hacia mediados del siglo XIV edificó un molino nuevo ${ }^{57}$.

Nuestro conocimiento del caso de Alcocer nos permite afirmar que a lo largo del siglo XIV subsistió cierta contribución de pequeños y medianos propietarios a la dotación de equipamientos hidráulicos, si bien ésta parece haberse realizado, en buena medida, en función de la dirección y de los intereses del gran propietario del lugar, el monasterio clariso de la villa. En efecto, en la primera mitad del siglo XIV, uno de los periodos de mayor rentabilidad relativa de los molinos, sabemos que las clarisas de Alcocer movilizaron la fuerza de trabajo de pequeños propietarios rurales, con frecuencia vinculados a la economía del monasterio (ya fuera como arrendatarios de tierra de las monjas, o como trabajadores por ellas contratados), para realizar tareas de reparación o mejora de molinos e infraestructuras hidráulicas asociadas, por medio de diversos contratos de arrendamiento. Sobre la conservación de la propiedad popular de dichos ingenios en este siglo, y más adelante, basta constatar que incluso en la documentación conservada en el archivo de las clarisas puede acreditarse sin dificultad ${ }^{58}$.

Sobre el régimen de copropiedad de los molinos, algunos testimonios permiten acreditar la actuación de estas modalidades asociativas en Alcocer: así por ejemplo, en 1285 la abadesa del monasterio clariso adquiere una novena parte de la propiedad del molino de Ordoño ${ }^{59}$, y en 1402 Juan Martínez consta como copropietario de los molinos de la Puente del Guadiela, donde tiene una de las tres ruedas del complejo ${ }^{60}$. En cambio, la documentación estudiada no da indicio alguno sobre eventuales conflictos entre particioneros, si bien esta omisión no parece particularmente significativa.

Por lo que se refiere al régimen de maquilas, sabemos por el fuero que en Alcocer los molinos maquilaban a 20, y de San Juan a San Miguel a 15, como regla. En Cifuentes, los molinos señoriales maquilaban a 20 por decisión de la primera señora de la villa, Mayor Guillén ${ }^{61}$, ratificada luego por su hija y heredera Beatriz de Portugal ${ }^{62}$. Al menos en una ocasión, en los molinos de la Puente del Guadiela, consta la presencia de un molinero contratado, un tal Alfonso Garrido, que no participa de la propiedad de los mismos, y que en consecuencia habría de percibir por su trabajo la cuarta parte de los derechos de maquila ${ }^{63}$.

57 SÁNCHez Benito: Las tierras de Cuenca y Huete, p. 109, nota 414.

58 Valgan como ejemplos: la casa de molinos en la Cuesta de Gárgoles que dona Gil Pérez de Atienza a las clarisas en 1335 (AHN, Clero, 567/16), y los molinos de la Puente del Guadiela cedidos por Juan Martínez a Constanza de Villena en 1402 (AHN, Clero, 568/9).

59 AHN, Clero, 566/17.

60 AHN, Clero, 568/9.

61 En 1260: Layna Serrano: Historia de Cifuentes, p. 268.

62 En 1272: AHN, Clero, 566/12-13.

63 La presencia de este molinero se detecta en los actos de toma de posesión de los molinos por parte del monasterio clariso, en 1429: AHN, Clero, 569/2. 


\section{IMPORTANCIA ECONÓMICA.}

Acerca de la importancia económica de los molinos cabe en primer lugar analizar la evolución de los precios de estos ingenios. Para las tierras de Cuenca y Huete, Aguadé recogió algunos precios desde finales del siglo XII y comienzos del XIII (medio molino más una tierra: 50 maravedíes; medio molino: 44 maravedíes; un tercio de molino: 14 maravedíes). Contrastando con estos precios tan modestos, encuentra un molino que en 1206 costó 400 maravedíes. A buen seguro, la importante diferencia en precio entre estos molinos se debe a las mayores complejidad y dimensiones del último mencionado, y a su pertenencia al espacio urbano de Cuenca ${ }^{64}$. Por su parte, para el conjunto del siglo XIV, Sánchez Benito ha observado unos precios notablemente más elevados (que van desde los 725 hasta los 7.000 maravedíes) ${ }^{65}$.

Por las características de la información contenida en la documentación que sirve de base a este estudio, no nos ha sido posible averiguar el precio de los molinos integrados en el patrimonio monástico de las clarisas de Alcocer. Tan sólo sabemos que en 1285 una novena parte del molino de Ordoño se vendió en 50 maravedíes ${ }^{66}$. Este dato nos permite realizar una estimación hipotética del valor total de dicho complejo molinero, para esa fecha, en 450 maravedíes, cifra que, con todo, parece modesta comparada con las más altas alcanzadas por molinos de Huete y Cuenca que dependían del espacio urbano. Para una fecha ligeramente más reciente (1297), cuenta Sánchez Benito con el dato de un complejo de molinos que costó 4.000 maravedíes, pero estando situado en la ciudad de Cuenca ${ }^{67}$. El molino de Ordoño, como perteneciente al ámbito rural, se mantenía en una escala de precios más modesta, si hemos de aceptar que la transacción de 1285 se realizó por un precio justo.

Por lo que toca a las rentas generadas por la explotación de los molinos, Aguadé recogió el dato de un molino que en 1206 rendía a sus propietarios 15 maravedíes al año ${ }^{68}$. Para el siglo XIV, Sánchez Benito añade datos de rentabilidad mucho más elevada, asociados a molinos más importantes, dependientes del medio urbano ${ }^{69}$. Sobre los molinos de las clarisas de Alcocer, poseemos algunos datos esparcidos sobre rentabilidad: en 1337, los molinos de Mari Cosida rentaban 12'5 maravedíes al año, y los de la Balsa de Cifuentes, 36 cahíces de trigo al año ${ }^{70}$; en 1438, los molinos de la Cuesta de Gárgoles de Arriba y los de la Fuente Rodera rentaban 7 cahíces (de la medida de Ávila, a seis fanegas el

\footnotetext{
64 Aguadé Nieto: «Notas para el estudio del molino», p. 76.

65 SÁNCHEZ BENITO: Las tierras de Cuenca y Huete, p. 107.

66 AHN, Sellos, 55/4.

67 SÁnChez Benito: Las tierras de Cuenca y Huete, p. 107.

68 Aguadé NiEto: «Notas para el estudio del molino», p. 77.

69 SÁnCHEZ BENITO: Las tierras de Cuenca y Huete, p. 112.

70 AHN, Clero, 567/17-18.
} 
cahíz) de trigo al año ${ }^{71}$; un censo enfitéutico se concertó en 1496 sobre el molino de aceite de Alcocer, por un pago anual de 2'5 cántaros de aceite ${ }^{72}$. Como observamos, los datos conocidos de rentabilidad de los molinos del monasterio dibujan un panorama modesto, con cierta propensión de rentas expresadas en especie, como conviene a la escala de economía rural en que se insertan. Si esta muestra es suficientemente representativa, nos permitiría apuntalar la imagen que las anteriores descripciones proporcionan sobre estos molinos, pequeños y medianos, adaptados a las necesidades de una modesta economía rural, incapaces en consecuencia de generar rentabilidades elevadas.

Por otro lado, un aspecto particularmente relevante referido a la importancia económica de los aprovechamientos molineros en el Alcocer medieval atañe a la especial intensificación de la dedicación del monasterio a la adquisición y gestión de rentas de molinos, intensificación que se percibe con la mayor claridad en la primera mitad del siglo XIV, y que parece posible relacionar con las primeras manifestaciones de la incipiente crisis económica bajomedieval en la región. En efecto, en la primera mitad del siglo XIV, el monasterio clariso de Alcocer atraviesa una etapa decisiva. Luego de la definitiva desvinculación de la dinastía de la fundadora respecto de los destinos del monasterio, a partir de 1312 , la comunidad clarisa se halla en la necesidad de afirmar esquemas propios de gestión del patrimonio monástico de la institución, con independencia de las labores de protección y alta dirección que la familia de Guzmán había desempeñado hasta entonces en beneficio de las monjas. Se abre, en consecuencia, una nueva etapa en la vida del convento, caracterizada por la renovación de los presupuestos con arreglo a los cuales se toman las principales decisiones sobre la gestión del patrimonio monástico. La coincidencia de esta nueva etapa de independencia del convento en la gestión del patrimonio con la coyuntura de crisis económica dará lugar a esa insoslayable intensificación de la atención del convento hacia las rentas derivadas de la explotación de molinos a la que nos venimos refiriendo, como una de las direcciones fundamentales en que evoluciona el modelo patrimonial del monasterio.

En efecto, es precisamente en torno al cambio de siglo, y durante las primeras décadas del siglo XIV, cuando el monasterio clariso de Alcocer otorga la mayor atención al recurso que las rentas de molinos representaban dentro de su patrimonio. En buena medida, la intensificación de esta dedicación de las clarisas de Alcocer a los aprovechamientos molineros es un efecto y una manifestación de la crisis. Es sabido que, en las primeras fases de una coyuntura depresiva, y muy especialmente por efecto de la carestía provocada por años de malas cosechas, como al parecer fueron en Castilla los primeros del siglo XIV, hubo necesariamente de producirse un alza en los precios del grano, alza que sin duda afectó positivamente, a corto plazo, a los intereses de los rentistas que co-

\footnotetext{
71 AHN, Clero, legajo 1965.

72 AHN, Clero, legajo 1967.
} 
braban en especie, y muy particularmente, a la rentabilidad de los molinos. Con carácter general, la rentabilidad de los molinos evoluciona en función de la variable relación que en cada momento se establece entre los precios de los granos y los salarios de la mano de obra especializada encargada de su manejo. En el inicio de la crisis, el alza de los precios de los cereales se combinó por un tiempo con el mantenimiento de salarios relativamente bajos, propiciando un momento de extraordinaria rentabilidad para los molinos. En particular, Guy Bois ha caracterizado el periodo comprendido entre finales del siglo XIII y las primeras décadas del siglo XIV como la «edad de oro» de la rentabilidad de los molinos $^{73}$. En suelo hispano, esta impresión ha sido confirmada, entre otros, por Teófilo Ruiz para el caso de la tierra de Burgos, en que la rentabilidad de los molinos parece haberse elevado substancialmente a partir de la mitad del siglo XIII ${ }^{74}$. Por esta razón, parece correcto relacionar la importante atención que las clarisas de Alcocer dedican en esos años a la gestión de sus rentas de molinos con la previsible tendencia alcista de los precios del grano, y por ende, de la rentabilidad de los molinos, en el mismo periodo.

Por añadidura, existen razones de tipo social que apuntalan esta explicación meramente económica: parece que un rasgo común de la política de la época, en lo que toca a la dotación patrimonial de las comunidades religiosas femeninas, fue buscar para ellas rentas de fáciles gestión y cobro, que no obligaran a las monjas a intervenir en exceso, de suerte que se optaba por vincular preferentemente tierras y ganados al patrimonio de comunidades religiosas masculinas, en tanto que la asignación de rentas se estimaba más adecuada a las posibilidades de gestión que la clausura permitía a las monjas ${ }^{75}$. Teniendo en cuenta esta circunstancia, debe advertirse que la dedicación preferente a las rentas de molinos resultaba especialmente atractiva e idónea para un convento femenino, tanto por su alta rentabilidad, como por lo adecuado de su gestión a las posibilidades y necesidades de las monjas.

Como hemos visto, en 1323 las monjas encargaron al molinero de la localidad vecina de Valdeolivas la construcción de una presa y de un caz o canal de derivación de agua para dar servicio a unos molinos situados junto al monasterio $^{76}$. Esta política de intensificación de la atención a los aprovechamientos molineros se verá reforzada con dos importantes contratos de arrendamiento de molinos suscritos con el mismo molinero en $1328^{77}$ y $1331^{78}$, así como con otro

73 BoIs, Guy: Crise du féodalisme, París, 1976, pp. 205-211.

74 RuIz, Teófilo: Sociedad y poder real en Castilla (Burgos en la Baja Edad Media), Barcelona, 1981, pp. 71-94.

75 Sobre la conciencia que en la época existía acerca de la idoneidad de las rentas de molinos para las comunidades religiosas femeninas, cfr. AGUADÉ NIETO: «Mozarabismo y aculturación en la sociedad rural medieval castellana», en MORILLA CRITZ et alii (eds.), Impactos exteriores sobre el mundo rural mediterráneo, Madrid, 1997, pp. 225-266 [239-240].

${ }^{76}$ AHN, Clero, 567/7.

77 AHN, Clero, 567/11. 
contrato suscrito con el paniaguado Domingo Máñez de Beteta, para la construcción de una nueva casa de molinos, en $1327^{79}$, y parece razonable ponerla en relación, en atención a lo dicho, con la coyuntura económica del momento.

\section{CONCLUSIONES.}

De todo lo anterior cabe derivar algunas conclusiones de interés:

El caso particular de los molinos del monasterio de Santa Clara de Alcocer debe en todo momento ponerse en relación con la realidad general del molino hidráulico, que contribuye a ilustrar.

El recurso a las rentas de molinos pareció especialmente adaptado a las necesidades de una comunidad religiosa femenina, cuyas posibilidades de actividad económica se veían restringidas por efecto de la clausura, y en consecuencia constituyó uno de los pilares esenciales sobre los que se fundó y sostuvo el patrimonio monástico de la comunidad.

La etapa de mayor dedicación molinera por parte del monasterio clariso de Alcocer coincide con el periodo de mayor rentabilidad histórica de los molinos, entre mediados del siglo XIII y mediados del siglo XIV, respondiendo a las necesidades y a los esquemas de gestión autónoma del patrimonio monástico por parte de la comunidad conventual, una vez que la familia de la fundadora había desceñido los lazos de la protección que había venido dispensando al monasterio.

La participación del monasterio clariso de Alcocer como propietario de molinos en la economía de la zona parece haber sido de cierto relieve: cabe suponer verosímilmente que la comunidad se habrá contado entre los mayores propietarios de recursos molineros en la comarca.

Con todo, los datos conocidos sobre el tamaño, la rentabilidad, la complejidad técnica y las inversiones en estos molinos permiten afirmar que se trata en cualquier caso de dotaciones de infraestructura de importancia pequeña y mediana, propias de una economía de escala rural, sin alcanzar las dimensiones de las grandes concentraciones molineras de la cuenca del Júcar, o de las que se concentraban en torno a Cuenca.

\footnotetext{
78 AHN, Clero, 567/14.

79 AHN, Clero, 567/10.
} 